\title{
Congenital Absence of Left Atrial Appendage Diagnosed by Multimodality Imaging
}

\author{
Yoshinari Enomoto, ${ }^{1, *}$ MD, Go Hashimoto, ${ }^{1, *}$ MD, Naohiko Sahara, ${ }^{1, *}$ MD, Hikari Hashimoto, ${ }^{1}$ MD, \\ Hiroki Niikura, ${ }^{1}$ MD, Keijiro Nakamura, ${ }^{1}$ MD, Raisuke Iijima, ${ }^{1}$ MD, Hidehiko Hara, ${ }^{1}$ MD, \\ Makoto Suzuki, ${ }^{1} \mathrm{MD}$, Mahito Noro, ${ }^{1,2} \mathrm{MD}$, Masao Moroi, ${ }^{1} \mathrm{MD}$, \\ Kaoru Sugi, ${ }^{1,3}$ MD and Masato Nakamura, ${ }^{1}$ MD
}

\begin{abstract}
Summary
A 70-years-old male with a history of hypertension and drug resistant paroxysmal atrial fibrillation (AF) presented to our hospital for catheter ablation to his symptomatic AF. He had no prior surgical or percutaneous procedure to close or exclude the left atrial appendage (LAA). A transesophageal echocardiography (TEE) was performed to rule out intra-cardiac thrombus prior to the ablation procedure. Although the TEE imaging at multiple acquisition angles was obtained, the LAA could not be visualized and an absence of the LAA was suspected. An absence of the LAA was confirmed using cardiac computed tomography (CT), which included 3D reconstruction. Additionally, the LAA was not visualized with left atrium (LA) angiography. During the ablation procedure, 3D voltage mapping in LA was created and no low voltage area or abnormal potential was recorded around the usual root location of the LAA. Successful electrical pulmonary vein isolation was achieved with no major complications. After six months of follow-up, the patient remained in sinus rhythm without any antiarrhythmic drugs and showed no related clinical symptoms. He stopped his anticoagulation therapy due to lack of evidence of AF recurrence and an absence of LAA. Multimodality imaging allowed us to identify the congenital absence of LAA.
\end{abstract}

Key words: Atrial fibrillation, Thromboembolism, Catheter ablation

(Int Heart J 2018; 59: 439-442)

$\mathrm{T}$ he left atrial appendage (LAA) is one of the most common sites for thrombus formation in patients with atrial fibrillation (AF). Although several cases have been reported, ${ }^{1-4)}$ an absence of LAA is an extremely rare condition and it is likely a congenital anatomical variation. Herein, we report a rare case of congenital absence of LAA diagnosed by several cardiac imaging evaluations and also that could be obtained by the left atrial (LA) bipolar voltage map during the AF ablation procedure.

\section{Case Report}

A 70-years-old male with a history of hypertension and drug resistant paroxysmal AF was referred to our hospital for catheter ablation to his symptomatic AF. He was treated with bepridil $200 \mathrm{mg}$ /day for his rhythm control and rivaroxaban $15 \mathrm{mg} /$ day for stroke prophylaxis $\left(\mathrm{CHA}_{2}\right.$ $\mathrm{DS}_{2}$-VASc score; 2). He had no prior surgical or percutaneous procedure to close or exclude the LAA. A transesophageal echocardiography (TEE) was performed to rule out intra-cardiac thrombus prior to the ablation procedure. Although both two dimensional (2D) and threedimensional (3D) TEE imaging at multiple acquisition angles were obtained using an X7-2 matrix probe and iE 33 ultrasound system (Philips Medical Systems, Andover, MA), the LAA could not be visualized and an absence of the LAA was suspected (Figure 1A, B). An absence of the LAA was also confirmed by cardiac computed tomography $(\mathrm{CT}$ ), which included 3D reconstruction (Figure 1C, D). Both of these modalities showed no apparent intracardiac thrombus, and then he followed in having catheter ablation. Prior to pulmonary vein (PV) isolation, LA angiography was performed and the LAA was also not visualized around the usual root location of the LAA (Figure 2 A). Voltage map used with Ensite Navx system (St. Jude Medical Centre, St. Paul, MN) in the LA was created and no apparent low voltage area or abnormal potential were recorded around the usual root location of the LAA (Figure 2B). Successful electrical PV isolation was achieved with no major complications. No atrial arrhythmia and non-PV ectopic beats could be induced under the admini-

From the ${ }^{1}$ Division of Cardiovascular Medicine, Toho University Ohashi Medical Center, Tokyo, Japan, ${ }^{2}$ Division of Cardiovascular Center, Toho University Sakura Medical Center, Chiba, Japan and ${ }^{3}$ Division of Cardiology, Odawara Cardiovascular Hospital, Kanagawa, Japan.

*These authors contributed equally to this work.

Address for correspondence: Yoshinari Enomoto, MD, Division of Cardiovascular Medicine, Toho University Ohashi Medical Center, 2-17-6 Ohashi, Meguro-ku, Tokyo 153-8515, Japan. E-mail: yenomo1225@oha.toho-u.ac.jp

Received for publication February 17, 2017. Revised and accepted May 31, 2017.

Released in advance online on J-STAGE March 12, 2018.

doi: 10.1536/ihj.17-094

All rights reserved by the International Heart Journal Association. 


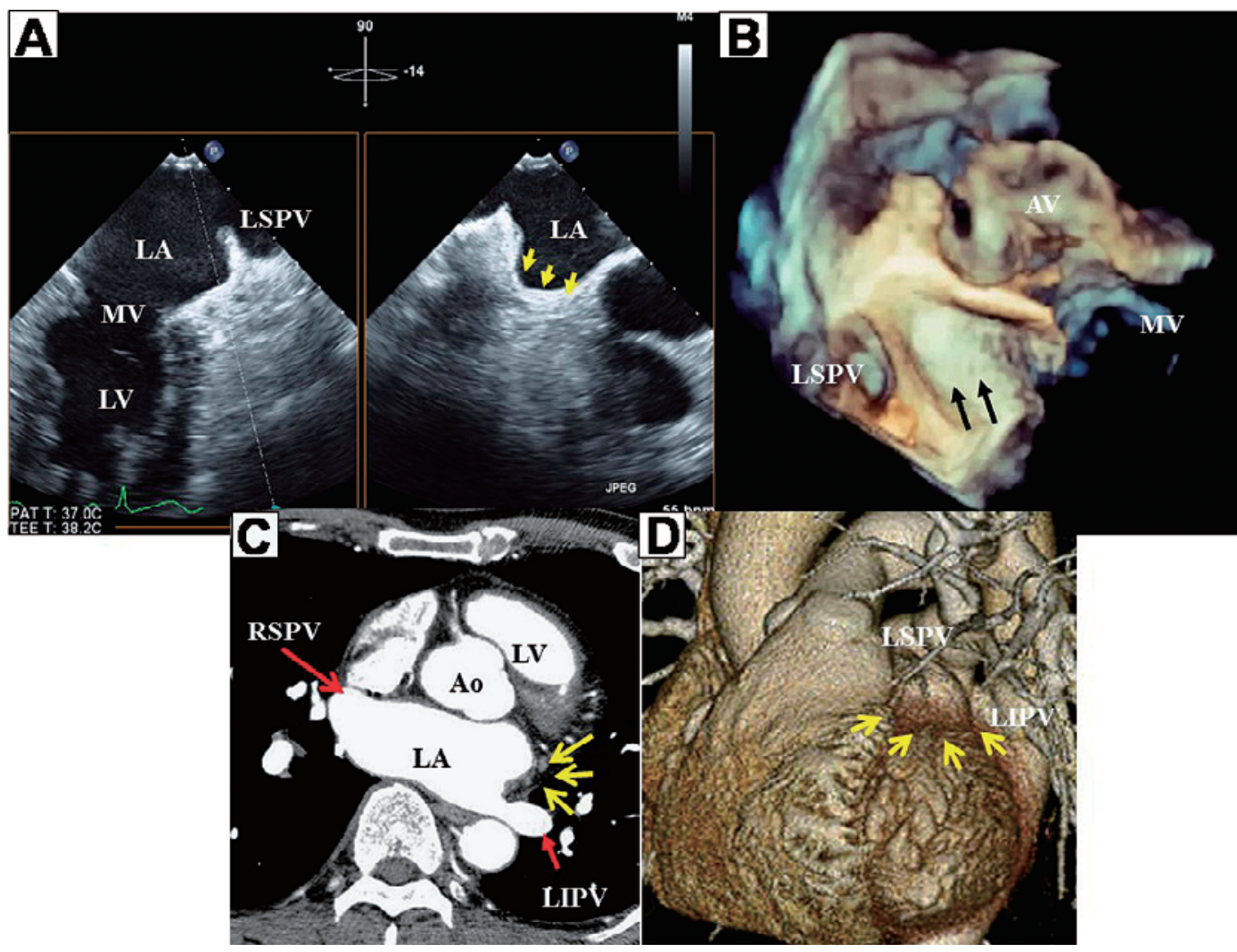

Figure 1. A: TEE imaging of the LA (mid esophageal level, 90 degrees) shows no evidence of the LAA (yellow arrows). B: Real-time 3D TEE demonstrates congenitally absent LAA (black arrows). C: Cardiac CT axial image of the LA depicting absence of LAA at its normal position (yellow arrows). D: On 3D volume-rendered CT image, yellow arrow points to the area where LAA would normally have been located. RSPV indicates right superior pulmonary vein; LIPV, left inferior pulmonary vein; LSPV, left superior pulmonary vein; LA, left atrium; LV, left ventricle; and Ao, Aorta

stration of isoproterenol and adenosine after the establishment of PV isolation. The procedure was deemed complete at this point. After six months of follow-up, the patient remained in sinus rhythm without the use of antiarrhythmic drugs and showed no related clinical symptoms. The patient stopped his oral anticoagulant due to lack of AF recurrence and an absence of LAA.

\section{Discussion}

Recently, the LAA has been of the significant interest because of its recognition as the trigger source of AF and development of the LAA closure device. ${ }^{5,6)}$ The LAA typically develops in the third week of embryonic life and is located within the pericardium. ${ }^{7)}$ The role of LAA includes producing a high level of atrial natriuretic factor and it contributes to the contractile function of the LA. ${ }^{7}$ Given that more than $90 \%$ of thrombus is identified in LAA in patients with non-valvular $\mathrm{AF}^{8)}$ surgical and percutaneous procedures for LAA exclusion have been developed for patients who are not candidates for long-term anticoagulation therapy (AT) or at high risk of thromboembolism. A TEE is often performed to ensure the absence of any LAA thrombus or describe detailed cardiac anatomy prior to the electrical cardioversion, AF ablation and cardiac surgery. Furthermore, Sakr, et al. reported that
LAA morphology evaluated by TEE may be useful for predicting ischemic cerebral stroke in patients with nonvalvular AF, especially in those patients with a low CHADS2 score.9) Taken together, the LAA evaluated by TEE is not only the most simple modality to detect the LAA thrombus but also the best modality to give us a much information on patients with non-valvular AF. Advanced sonographic techniques such as biplane and multiplane TEE allow one to visualize LAA in most cases. The differential diagnosis for non-visualization of the LAA during TEE imaging includes flush thrombus, variant anatomical features, poor echocar-diographic windows, prior surgical ligation, or insertion of an occlude device. ${ }^{1)}$ In the present case, although we tried to get images of LAA, we could not find any evidence of presence of LAA using several imaging modalities which included TEE, CT, and LA angiography.

The notable finding of our case and what differentiates our study from other reports is that we recorded the LA bipolar voltage map using a 3D mapping system. Electro-anatomical voltage mapping has the potential to identify the atrial scar area which may play a key role in the AF substrate. Although the etiology of atrial scar is not fully elucidated, previous reports have showed that LA scar is an independent predictor of long-term recurrence. ${ }^{10)}$ The other congenital heart diseases, such as atrial septal 


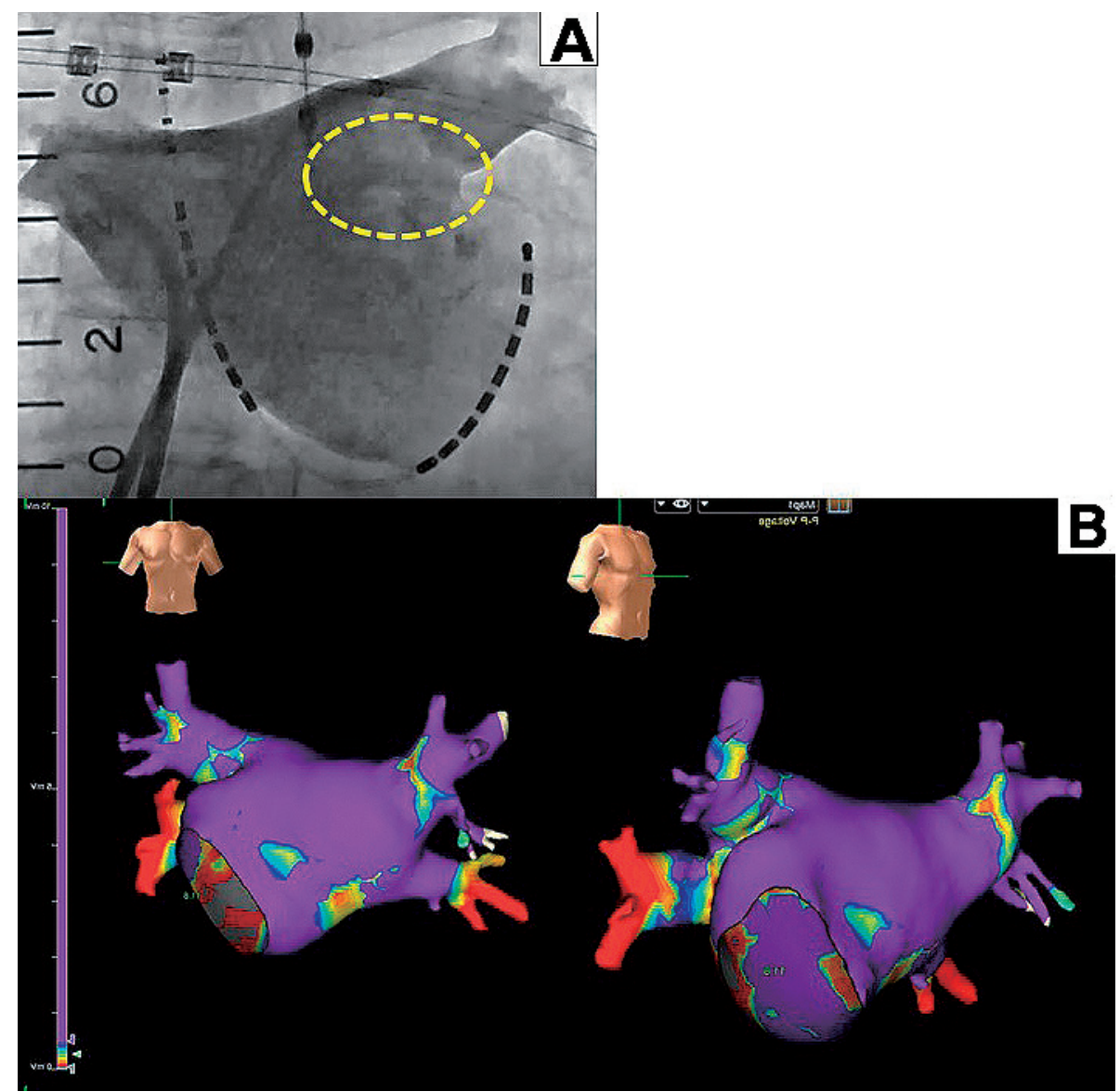

Figure 2. A: Left atrial angiography. The LAA was not visualized on the left atrial angiography when a pre-shaped 8-Fr sheath (SL1; St. Jude Medical, St. Paul, MN) was placed near the orifice of left superior pulmonary vein. (Anterior-posterior view, yellow broken line.) B: The 3D electrical bipolar voltage map in the LA projected in the anterior-posterior view (right panel) and right anterior oblique view (left panel). Lowvoltage area defined as $<0.5 \mathrm{mV}$.

defect (ASD), have more atrial scar area compared with normal heart ${ }^{11)}$, whereas the LA voltage map in the present case did not show any abnormal potential or low voltage area including the usual root location of the LAA. Of course, although the volume or pressure load to LA is different from ASD, this finding may suggest that the absence of LAA is a "benign congenital variation" in terms of electrophysiological characteristics. To the best of our knowledge, this is the first case report that also describes the LA bipolar voltage map with congenital absence of LAA.

Because of the absence of LAA and that the patient remained in sinus rhythm without any evidence of AF recurrence, the patient stopped his AT. Theoretically, in patients with congenital absence of LAA, this may be equivalent to the condition of those patients who are undergoing LAA exclusion, ${ }^{4}$ therefore AT is not mandatory in terms of thromboembolism prophylaxis. Further reports are warranted to determine if long-term AT can be waived in $\mathrm{AF}$ patients with absence of the LAA.

In conclusion, we report the absence of LAA diagnosed by multimodality imaging and also describe the normal LA voltage map which implies the absence of
LAA as a benign variation.

\section{Disclosures}

Conflicts of interest: None.

\section{References}

1. Collier P, Cavalcante JL, Phelan D, et al. Congenital absence of the left atrial appendage. Circ Cardiovasc Imaging 2012; 5: 549-50.

2. Di Gioia G, Mega S, Visconti S, et al. Congenital absence of left atrial appendage in a patient with intracranial hemorrhage. Am J Case Rep 2015; 16: 514-6.

3. Zhang ZJ, Dong JZ, Ma CS. Congenital absence of the left atrial appendage: a rare anatomical variation with clinical significance. Acta Cardiol 2013; 68: 325-7.

4. Saleh M, Balakrishnan R, Kontak LC, et al. Congenital absence of the left atrial appendage visualized by 3D echocardiography in two adult patients. Echocardiography 2015; 32: 1206-10.

5. Di Biase L, Burkhardt JD, Mohanty $\mathrm{P}$, et al. Left atrial appendage isolation in patients with longstanding persistent $\mathrm{AF}$ undergoing catheter ablation: BELIEF trial. J Am Coll Cardiol 2016; 68: $1929-40$ 
6. Reddy VY, Doshi SK, Sievert H, et al. Percutaneous left atrial appendage closure for stroke prophylaxis in patients with atria fibrillation 2.3-year follow-up of the PROTECT AF (Watchman left atrial appendage system for embolic protection in patients with atrial fibrillation) trial. Circulation 2013; 127: 720-9.

7. Al-Saady NM, Obel OA, Camm AJ. Left atrial appendage: Structure, function, and role in thromboembolism. Heart 1999; 82: 547-55.

8. Stoddard MF, Dawkins PR, Prince CR, Ammash NM. Left atrial appendage thrombus is not uncommon in patients with acute atrial fibrillation and a recent embolic event: a transesophageal echocardiographic study. J Am Coll Cardiol 1995; 25: 452-9.
9. Sakr SA, El-Rasheedy WA, Ramadan MM, El-Menshawy I, Mahfouz E, Bayoumi M. Association between left atrial appendage morphology evaluated by trans-esophageal echocardiography and ischemic cerebral stroke in patients with atrial fibrillation. Int Heart J 2015; 56: 329-34.

10. Verma A, Wazni OM, Marrouche NF, et al. Pre-existent left atrial scarring in patients undergoing pulmonary vein antrum isolation: an independent predictor of procedural failure. J Am Coll Cardiol 2005; 18:45: 285-92.

11. Roberts-Thomson KC, John B, Worthley SG, et al. Left atrial remodeling in patients with atrial septal defects. Heart Rhythm 2009; 6: 1000-6. 